\title{
HOLOMORPHIC SPHERES IN LOOP GROUPS AND BOTT PERIODICITY*
}

\author{
RALPH L. COHEN ${ }^{\dagger}$, ERNESTO LUPERCIO $\ddagger$, AND GRAEME B. SEGAL ${ }^{\S}$
}

\begin{abstract}
In this paper we study the topology of spaces of holomorphic maps from the Riemann sphere $\mathbb{P}^{1}$ to infinite dimensional Grassmanian manifolds and to loop groups. Included in this study is a complete identification of the homotopy types of $\mathrm{Hol}_{k}\left(\mathbb{P}^{1}, B U(n)\right)$ and of $H_{o l}\left(\mathbb{P}^{1}, \Omega U\right)$, where the subscript $k$ denotes the degree of the map. These spaces are shown to be homotopy equivalent to the $k^{t h}$ Mitchell - Segal algebraic filtration of the loop group $\Omega U(n)$ [7], and to $B U(k)$, respectively.
\end{abstract}

Introduction. One of the most important theorems in Topology and Geometry is the "Bott Periodicity Theorem". In its most basic form it states that there is a natural homotopy equivalence,

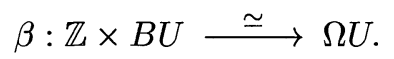

Here $U$ is the infinite unitary group, $U=\varliminf_{n} U(n), B U=\varliminf_{n} B U(n)$ is the limit of the classifying spaces, and $\Omega U=C^{\infty}\left(\overrightarrow{S^{1}}, U\right)$ is the space of smooth, basepoint preserving loops. Here and throughout the rest of this paper all spaces will assumed to be equipped with a basepoint, and all maps and mapping spaces will be basepoint preserving.

If we input the fact that $U \simeq \Omega B U$, Bott periodicitiy states that there is a natural homotopy equivalence

$$
\beta: \mathbb{Z} \times B U \stackrel{\simeq}{\longrightarrow} \Omega^{2} B U=C^{\infty}\left(S^{2}, B U\right) .
$$

In a paper which first pointed to the deep relationship between the index theory of Fredholm operators and Algebraic Topology, Atiyah [1], defined a homotopy inverse to the Bott map $\beta$, which can be viewed as a map

$$
\bar{\partial}: C^{\infty}\left(S^{2}, B U\right) \stackrel{\simeq}{\longrightarrow} \times B U .
$$

This map was defined by studying the index of the family of operators obtained by coupling the $\bar{\partial}$ operator to a smooth map from $S^{2}$ to a Grassmannian.

Since the mapping spaces $C^{\infty}\left(S^{2}, B U\right)$ and $\Omega U$ both have path components naturally identified with the integers, we denote by $C^{\infty}\left(S^{2}, B U\right)_{k}$ the path components consisting of degree $k$ - maps. Thus Bott periodicity, together with Atiyah's results says that for each integer $k$, there is a natural homotopy equivalence,

$$
\bar{\partial}: C^{\infty}\left(S^{2}, B U\right)_{k} \stackrel{\simeq}{\longrightarrow} B U .
$$

The goal of this paper is to prove a holomorphic version of this result. We first note however that the homotopy type of $B U$ has many different models, several of which carry a holomorphic structure. For the purposes of this paper we think of $B U$

\footnotetext{
${ }^{*}$ Received July 30, 1999, accepted for publication January 10, 2000.

†Dept. of Mathematics, Stanford University, Stanford, California 94305, USA (ralph@math. stanford.edu). The research of this author was partially supported by a grant from the NSF.

$\ddagger$ Department of Mathematics, University of Michigan, Ann Arbor, Michigan 48109, USA (lupercio @math.lsa.umich.edu). The research of this author received partial support from CONACYT.

$\S$ Dept. of Pure Mathematics and Mathematical Statistics, Cambridge University, Cambridge, England (G.B.Segal@dpmms.cam.ac.uk).
} 
as the colimit of the finite dimensional Grassmannians $G r_{m}\left(\mathbb{C}^{n}\right)$ of $m$ - dimensional subspaces of $\mathbb{C}^{n}$. We define $\operatorname{Hol}_{k}\left(\mathbb{P}^{1}, B U\right)$ to be $\lim _{m, n} H o l_{k}\left(\mathbb{P}^{1}, G r_{m}\left(\mathbb{C}^{n}\right)\right)$ topologized as a subspace of $C^{\infty}\left(S^{2}, B U\right)=\varliminf_{m, n} C^{\infty}\left(S^{2}, G r_{m}\left(\mathbb{C}^{n}\right)\right)$, where these mapping spaces are given the compact open topology.

The following is the first result of this paper.

THEOREM 1. For each positive integer $k$, there is a natural homotopy equivalence

$$
H_{o l}\left(\mathbb{P}^{1}, B U\right) \stackrel{\simeq}{\longrightarrow} B U(k) .
$$

REMARK. Observe that this theorem states implies the inclusion of holomorphic maps into all smooth maps (of degree $k$ ),

$$
H_{o l}\left(\mathbb{P}^{1}, B U\right) \hookrightarrow C^{\infty}\left(S^{2}, B U\right)_{k}
$$

is homotopy equivalent to the inclusion of classifying spaces,

$$
B U(k) \hookrightarrow B U .
$$

In future work we will study consequences of this theorem to the understanding of holomorphic $K$ - theory of a smooth, projective variety.

Our other main result in this paper has to do with an identification of the topology of subspaces $H_{o l}\left(\mathbb{P}^{1}, B U(n)\right)$ of $H_{o l}\left(\mathbb{P}^{1}, B U\right)$. By theorem 1, this latter space is homotopy equivalent to $B U(k)$, and so the inclusion $B U(n) \subset B U$ induces a map

$$
j: H_{o l}\left(\mathbb{P}^{1}, B U(n)\right) \rightarrow B U(k) .
$$

On the other hand, the inclusion of holomorphic maps into smooth maps, together with the identification of $\Omega^{2} B U(n)$ with $\Omega U(n)$ induces a map

$$
\iota: \operatorname{Hol}_{k}\left(\mathbb{P}^{1}, B U(n)\right) \rightarrow \Omega_{k} U(n) .
$$

Furthermore, it is not difficult to see that the maps $j$ and $\iota$ are compatible when composed to $B U \simeq \Omega U$.

Now S. Mitchell in [7] described an algebraic filtration of the loop group $\Omega S U(n)$ by compact, complex subvarieties:

$$
F_{1, n} \subset F_{2, n} \subset \cdots \subset F_{k, n} \subset \cdots \subset F_{\infty, n} \simeq \Omega S U(n) .
$$

In this filtration, $F_{1, n}=\mathbb{C P}^{n-1}$ included in $\Omega U(n)$ via the usual complex $J$ - map. $F_{k, n} \subset \Omega U(n)$ is the set of all $k$ - fold products of elements of $F_{1, n} \subset \Omega U(n)$. See [11]. The subspaces $F_{k, n}$ have as their homology, precisely the intersection of $H_{*}(B U(k))$ and $H_{*}(\Omega S U(n))$, viewed as subgroups of $H_{*}(B U)=H_{*}(\Omega S U)$. In [8], Richter proved that this filtration stably splits, so that the loop group $\Omega S U(n)$ is stably homotopy equivalent to a wedge of the subquotients, $F_{k+1, n} / F_{k, n}$. The following theorem was conjectured by Mann and Milgram in [6] after an analysis of the holomorphic mapping spaces $\operatorname{Hol}_{k}\left(\mathbb{P}^{1}, G r_{m}\left(\mathbb{C}^{n}\right)\right)$ :

THEOREM 2. There is a natural homotopy equivalence

$$
\operatorname{Hol}_{k}\left(\mathbb{P}^{1}, B U(n)\right) \simeq F_{k, n} .
$$


These theorems are proven by using the identification of the loop group $\Omega U(n)$ with a certain moduli space of holomorphic bundles over $\mathbb{P}^{1}$, together with holomorphic trivializations on a disk. This identification was established in [9]. We then identify the homotopy type of $\mathrm{Hol}_{k}\left(\mathbb{P}^{1}, B U(n)\right)$ with a subspace of this moduli space consisting of bundles that are "negative" in the sense that they are (holomorphically) isomorphic to a direct sum of line bundles, each of which has nonpositive first Chern class. The topology of these moduli spaces are then studied in two ways: homologically, using calcluations of [6], and Morse theoretically, by analyzing the gradient flow of the Dirichlet energy functional on $\Omega U(n)$.

This paper is organized as follows. In section 1 we review some results from the theory of loop groups. The main reference for this material is Pressley and Segal's book [9]. This theory will in particular allow us to define the terms and maps in the statements of the above theorems more carefully. In section 2 we give a proof of theorems 1 and 2, modulo a technical argument establishing that certain maps are (quasi)fibrations. This argument is carried out in section 3.

The real Bott periodicity analogues of the above theorems (where the unitary groups are replaced by orthogonal and symplectic groups) were established in the Stanford University Ph.D thesis of the second author written under the direction of the first author. The authors are grateful to Paulo Lima-Filho, Steve Mitchell, Paul Norbury, and Giorgio Valli for helpful conversations regarding this work.

1. Loop Groups. In this section we recall some of the basic constructions from the theory of loop groups as developed in [9]. We will use these constructions to define the holomorphic structures necessary to define the spaces and maps in the theorems described in the introduction. In what follows we will work with the Lie groups $U(n)$, but everything we use has obvious analogues for arbitrary compact semi-simple Lie groups. Again, we refer the reader to [9] for details.

As defined in the introduction, let $L G$ denote the space of smooth maps from the circle $S^{1}$ to a Lie group $G$. The loop group $L G L(n, \mathbb{C})$ has the following important subgroups.

1. The group $L^{+} G L(n, \mathbb{C})$ of maps $\gamma: S^{1} \rightarrow G L(n, \mathbb{C})$ that extend to holomorphic maps of the closed disk $D^{2} \rightarrow G L(n, \mathbb{C})$.

2. the group $L_{p o l} G L(n, \mathbb{C})$ of loops whose matrix entries are finite Laurent polynomials in $z$. That is, loops $\gamma$ of the form

$$
\gamma(z)=\sum_{k=-N}^{N} A_{k} z^{k}
$$

for some $N$, where the $A_{k}$ 's are $n \times n$ matrices.

3 . The based loop group $\Omega G L(n, \mathbb{C})$, and the corresponding subgroup of polynomial loops, $\Omega_{p o l} G L(n, \mathbb{C})$.

In the theory of loop groups, there is an underlying Hilbert space $H^{n}$ defined to be the space of square integrable functions from the circle to $\mathbb{C}^{n}$ :

$$
H^{n}=L^{2}\left(S^{1}, \mathbb{C}^{n}\right)
$$

Notice that this Hilbert space has a natural polarization:

$$
H^{n} \cong H_{+}^{n} \oplus H_{-}^{n}
$$


where $H_{+}^{n}$ consists of those functions whose negative Fourier coefficients are all zero; or equivalently those functions $f: S^{1} \rightarrow \mathbb{C}^{n}$ that extend to a holomorphic map of the disk.

Observe that the loop group $L G L(n, \mathbb{C})$ has a natural representation on the Hilbert space $H^{n}=L^{2}\left(S^{1}, \mathbb{C}^{n}\right)$ given by matrix multiplication. In particular the Laurent polynomial ring $\mathbb{C}\left[z, z^{-1}\right]$ acts on $H^{n}$, via the action of the loops

$$
z^{k} \rightarrow z^{k} \cdot I_{n \times n} \in L G L(n, \mathbb{C}) .
$$

Now recall the "restricted Grassmannian" $G r\left(H^{n}\right)$ of $H^{n}$ as defined in [9]. This is the space of all closed subspaces $W \subset H^{n}$ such that the orthogonal projections $p r_{+}: W \rightarrow H_{+}^{n}$ and $p r_{-}: W \rightarrow H_{-}^{n}$ are Fredholm and Hilbert-Schmidt operators respectively.

Let $G r_{\infty}\left(H^{n}\right) \subset G r\left(H^{n}\right)$ be the dense submanifold consisting of elements [W] $\in$ $G r\left(H^{n}\right)$ such that the images of the projections $p r_{+}: W \rightarrow H_{+}^{n}$ and $p r_{-}: W \rightarrow H_{-}^{n}$ consist of smooth functions. (See [9] $\$ 7.2$.)

For ease of notation we denote $G r_{\infty}\left(H^{n}\right)$ by $G r$. Consider the following important submanifolds of $\mathrm{Gr}$.

1. $G r_{0}=\left\{W \in G r: \exists k \geq 0\right.$ such that $\left.z^{k} H_{+} \subset W \subset z^{-k} H_{+}^{n}\right\}$

2. $G r^{(n)}=\{W \in G r: z W \subset W\}$

3. $G r_{0}^{(n)}=G r^{(n)} \cap G r_{0}$.

The action of $L G L(n, \mathbb{C})$ on $H^{n}$ induces an action on $G r\left(H^{n}\right)$, and it is proved in [9] that the orbit of $H_{+}^{n}$ is precisely $G r^{(n)}$. This is also its orbit under the subgroup $L U(n)$. The isotropy group of $H_{+}^{n}$ in $L G L(n, \mathbb{C})$ consists of those loops whose Fourier expansions contain only nonnegative powers of $z$. These loops are exactly the boundary values of holomorphic maps of the disk. In the above notation we called this subgroup $L^{+} G L(n, \mathbb{C})$. In fact there is a homeomorphism

$$
L G L(n, \mathbb{C}) / L^{+} G L(n, \mathbb{C}) \cong G r^{(n)} .
$$

Furthermore, if one restricts the action to $L U(n)$, one sees that the isotropy subgroup of $H_{+}^{n}$ is given by the subgroup $L U(n) \cap L^{+} G L(n, \mathbb{C})$. This is the subgroup of loops of $U(n)$ that are boundary values of holomorphic maps of the disk to $G L(n, \mathbb{C})$. A generalization of the maximum modulus principle, as proved in [9] shows that this subgroup consists only of the constant loops, $U(n) \subset L U(n)$. This, together with the analogous argument using $G r_{0}^{(n)}$ and polynomial loops proves the following.

THEOREM 1.1. There are homeomorphisms

$$
\begin{aligned}
\Omega U(n) \cong L U(n) / U(n) \cong L G L(n, \mathbb{C}) / L^{+} G L(n, \mathbb{C}) & \cong G r^{(n)} \\
\Omega_{p o l} U(n) \cong L_{p o l} U(n) / U(n) \cong L_{p o l} G L(n, \mathbb{C}) / L_{p o l}^{+} G L(n, \mathbb{C}) & \cong G r_{0}^{(n)} \\
\text { given by } \gamma & \rightarrow \gamma H_{+}^{n}
\end{aligned}
$$

These homeomorphisms determine the complex structure on the infinite dimensional manifolds $\Omega U(n)$ and $\Omega_{p o l} U(n)$ that are used in the statement of theorem 1 in the introduction. 
Given an element $W \in G r^{n}$ define the virtual dimension of $W, v d(W)$, to be the Fredholm index of the projection of the projection map

$$
p r_{+}: W \rightarrow H_{+}^{n}
$$

So in particular we have

$$
v d(W)=\operatorname{dim}\left(W \cap H_{-}^{n}\right)-\operatorname{dim}\left(W^{\perp} \cap H_{+}^{n}\right) .
$$

As we will see below, much of the analysis necessary to prove theorem 1 comes from studying the situation when the projection operator $p r_{+}: W \rightarrow H_{+}^{n}$ has trivial kernel, and hence $v d(W)=-\operatorname{dim}\left(\operatorname{coker}\left(p r_{+}\right)\right)$.

Now notice that since every element $W \in G r_{0}$ has the property that $z^{k} H_{+}^{n} \subset W \subset$ $z^{-k} H_{+}^{n}$ for some $k$, and hence by considering the projection $\bar{W} \subset z^{-k} H_{+}^{n} / z^{k} H_{+}^{n}$, one has that $G r_{0}$ is the union of finite dimensional Grassmannians,

$$
G r_{0}=\bigcup_{p} G r\left(z^{-p} H_{+}^{n} / z^{p} H_{+}^{n}\right) .
$$

and is therefore topologically equivalent to $\mathbb{Z} \times B U$. The integer denotes the virtual dimension of $W$.

Via the identification of $G r^{(n)}$ with the loop group, the virtual dimension determines the path component of the loop. In pariticular if $w d g(\gamma)$ denotes the winding number of the determinant of a loop in $U(n)$, then a straightforward exercise proves the following.

Proposition 1.1. Let $\gamma \in \Omega U(n)$ correspond to $W \in G r^{(n)}$. That is, $W=\gamma H_{+}^{n}$. Then the virtual dimension of $W$ and the winding number of $\gamma$ are related by

$$
v \cdot d(W)=-w d g(\gamma)
$$

Notice furthermore that if $\gamma \in \Omega_{p o l} U(n)$ is a polynomial loop, then this composite $\operatorname{det} \circ \gamma$ is also a polynomial loop in $S^{1} \in \mathbb{C}$. The only such polynomial maps (i.e polynomials in one variable of constant unit length) are $z \rightarrow z^{k}$ for some $k \in \mathbb{Z}$. Therefore we may conclude that the polynomial loop group of $S U(n)$ consists precisely of those polynomial loops in $\Omega_{p o l} U(n)$ with winding number zero. We again refer the reader to [9] for details.

Recall that there was another description of the loop group $\Omega U(n)$ in [9] that is given in terms of holomorphic bundles over the Riemann sphere $\mathbb{P}^{1}=\mathbb{C} \cup \infty=$ $D_{0} \cup D_{\infty}$, where $D_{0}=\{z:|z| \leq 1\}$ and $D_{\infty}=\{z:|z| \geq 1\}$.

Proposition 1.2. Let $\mathcal{C}_{k, n}$ denote the space $\mathcal{C}_{k, n}=\{$ isomorphism classes of pairs $(E, \phi)$, where $E \rightarrow \mathbb{P}^{1}$ is an $n$ - dimensional bundle of Chern class $c_{1}(E)=-k$, and $\phi$ is a holomorphic framing of $\left.E_{\left.\right|_{D_{\infty}}}\right\}$. Then there is a natural homeomorphism

$$
\mathcal{C}_{k, n} \stackrel{\cong}{\longrightarrow} \Omega_{k} U(n) \text {. }
$$

The homeomorphism in this proposition can be described as follows. Let $(E, \phi) \in$ $\mathcal{C}_{k, n}$. So $\phi:\left.E\right|_{D_{\infty}} \rightarrow \mathbb{C}^{n}$ is a fixed trivialization. Let $\sigma:\left.E\right|_{D_{0}} \rightarrow \mathbb{C}^{n}$ be any 
holomorphic trivialization of the restriction of $E$ to the other disk, $D_{0}$. On the intersection $S^{1}=D_{0} \cap D_{\infty}$, the trivializations $\phi$ and $\sigma$ differ by a loop $\gamma \in L G L(n, \mathbb{C})$. Of course the loop $\gamma$ depends on the choice of trivialization $\sigma$, but if a different trivialization, say $\tilde{\sigma}$ were used to construct a different loop, say $\tilde{\gamma}$, then $\gamma \cdot(\tilde{\gamma})^{-1}$ would be a loop that extends to a holomorphic map $\sigma \cdot(\tilde{\sigma})^{-1}: D_{0} \rightarrow G L(n, \mathbb{C})$. Thus $\gamma \cdot(\tilde{\gamma})^{-1} \in L^{+} G L(n, \mathbb{C})$. This procedure therefore gives a well defined map

$$
\mathcal{C}_{k, n} \rightarrow L G L(n, \mathbb{C}) / L^{+} G L(n, \mathbb{C}) \cong \Omega U(n) .
$$

We refer the reader to [9] for details of the argument proving that this map is a homeomorphism.

We note that with respect to the diffeomorphisms

$$
\mathcal{C}_{k, n} \cong \Omega_{k} U(n) \cong\left(G r^{(n)}\right)_{k}
$$

the Chern class of the bundle in $\mathcal{C}_{k, n}$ corresponds to the virtual dimension of the subspace $W \in G r^{(n)}$, which, as observed above, corresponds to the opposite of the winding number of the loop in $\Omega U(n)$.

These models of the loop groups will prove very important in our study of holomorphic mapping spaces. One aspect of these models that is quite useful is that they come equipped with filtrations by algebraic subvarieties. That is, there is a sequence of compact, complex subvarieties:

$$
F_{1, n} \hookrightarrow \cdots F_{k, n} \hookrightarrow F_{k+1, n} \hookrightarrow \cdots F_{\infty, n}=\Omega_{p o l} S U(n) \simeq \Omega S U(n) .
$$

The filtration $F_{k, n}$ can be defined as follows. As in [11], define the sub - semigroup $\tilde{\Omega}_{n}$ of the space of polynomial loops $\Omega_{p o l} U(n)$ to consist of loops that only involve non-negative powers of $z$ in their Fourier expansions. Furthermore, we write

$$
\tilde{\Omega}_{n}=\coprod_{k \geq 0} \tilde{\Omega}_{k, n}
$$

where $\tilde{\Omega}_{k, n}$ consists of loops of winding number $k$.

The space $F_{k, n}$ is homeomorphic to $\tilde{\Omega}_{k, n}$, but in order to see how these spaces give a filtration of $\Omega S U(n)$, we consider the loop

$$
\begin{aligned}
\lambda: S^{1} & \rightarrow U(n) \\
z & \rightarrow\left(\begin{array}{cccc}
z^{-1} & 0 & \ldots & 0 \\
0 & 1 & 0 & \ldots \\
0 & 0 & 1 & \ldots \\
& & \ldots & \\
& & & 1
\end{array}\right)
\end{aligned}
$$

Clearly $\lambda$ is a polynomial loop of winding number -1 . Therefore if we define

$$
F_{k, n}=\lambda^{k} \tilde{\Omega}_{k, n}
$$

then $F_{k, n}$ consists of polynomial loops of winding number zero. That is, $F_{k, n} \subset$ $\Omega_{p o l} S U(n)$. Moreover $F_{k, n} \subset F_{k+1, n}$ and

$$
\Omega_{p o l} S U(n)=\bigcup_{k \geq 1} F_{k, n}
$$


(See [7], [11].)

The homotopy theory of these filtrations has been studied in great detail by Mitchell [7], and by Richter in [8]. In particular one has the following properties (see [7])

Proposition 1.3. If one takes the limit over the rank $n$, then there is a natural homotopy equivalence

$$
\lim _{n \rightarrow \infty} F_{k, n} \simeq B U(k)
$$

Moreover the following diagram homotopy commutes:

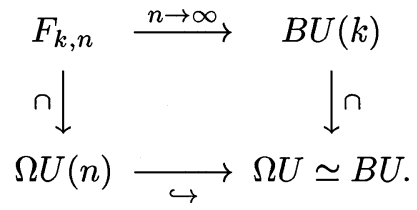

Furthermore the homology of this filtration is given by

$$
H_{*}\left(F_{k, n}\right) \cong H_{*}(\Omega U(n)) \cap H_{*}(B U(k)) \subset H_{*}(B U) .
$$

In [8] Richter also showed that this filtration stably splits. That is, there is a stable homotopy equivalence

$$
\Omega S U(n) \simeq \bigvee_{k} F_{k, n} / F_{k-1, n}
$$

Now the spaces of loops $\tilde{\Omega}_{k, n}$ can also be interpreted in the above Grassmannian models in the following way. Since $\gamma \in \tilde{\Omega}_{k, n}$ has only positive terms in its Fourier expansion, the space $W=\gamma H_{+}^{n}$ is a subspace of $H_{+}^{n}$. Moreover since $\gamma$ has winding number $k$, the subspace $W$ has virtual dimension $-k$, (proposition 1.2). This means that the dimension of $H_{+}^{n} / W$ is equal to $k$. Thus we have the following.

Proposition 1.4. Under the diffeomorphism described in theorem 1.1, we have that

$$
\begin{aligned}
\tilde{\Omega}_{k, n} & =\left\{W \in G r_{0}^{(n)}: W \subset H_{+}^{n} \text { anddim } H_{+}^{n} / W=k\right\} \\
& =\left\{W \in G r_{0}^{(n)}: v d(W)=-k, \text { and } \operatorname{ker}\left(p r_{+}: W \rightarrow H_{+}^{n}\right)=0\right\} .
\end{aligned}
$$

It will be helpful to have a description of $\tilde{\Omega}_{k, n}$ (or equivalently $F_{k, n}$ ) in terms of holomorphic bundles as well. To do this we need to introduce the notion of a negative holomorphic bundle.

By a well known theorem of Grothendieck [4], every holomorphic bundle over $\mathbb{P}^{1}$ is isomorphic to a direct sum of holomorphic line bundles of the form $O(j)$, where $c_{1}(O(j))=j \in \mathbb{Z}$. Furthermore this direct sum decomposition is unique (up to order). A holomorphic bundle $E \rightarrow \mathbb{P}^{1}$ is negative if

$$
E \cong O\left(j_{i}\right) \oplus \cdots \oplus O\left(j_{m}\right)
$$


with each $j_{i} \leq 0$. Notice that the dimension of $E$ is $m$, and the first Chern class is given by

$$
c_{1}(E)=\sum_{i=1}^{m} j_{i}
$$

It is a standard exercise that a holomorphic bundle $E$ over $\mathbb{P}^{1}$ is negative if and only if it can be holomorphically embedded into a trivial bundle. Such bundles are obtained by pulling back the universal bundle over a Grassmannian via a holomorphic map. A positive holomorphic bundle over $\mathbb{P}^{1}$ has the analogous definition, and this property corresponds to a bundle being generated by its holomorphic sections.

Define the sub - moduli space

$$
\mathcal{C}_{k, n}^{-} \subset \mathcal{C}_{k, n}
$$

to consist of those $(E, \phi) \in \mathcal{C}_{k, n}$ such that $E$ is negative.

Now an easy exercise verifies that with respect to the homeomorphism $\mathcal{C}_{k, n} \cong$ $G r^{(n)}$ described above, a holomorphic bundle $E$ being negative corresponds to the projection operator $p r_{+}: W \rightarrow H_{+}^{n}$ having zero kernel, and so $v d(W)=-\operatorname{dimcoker}\left(p r_{+}\right)$. By proposition 1.3 we therefore have the following.

Proposition 1.5. With respect to the diffeomorphisms $\mathcal{C}_{k, n} \cong \Omega_{k} U(n) \cong\left(G r^{(n)}\right)_{k}$, we have that

$$
\begin{aligned}
\tilde{\Omega}_{k, n} & \cong \mathcal{C}_{k, n}^{-} \cap \Omega_{p o l} U(n) \\
& \cong \mathcal{C}_{k, n}^{-} \cap G r_{0}^{(n)} .
\end{aligned}
$$

2. Proofs of Theorems 1 and 2. In this section we give proofs of theorems 1 and 2, modulo a technical lemma whose proof we delay until the next section. We begin with theorem 2 , which asserts that $F_{k, n}$ is homotopy equivalent to the space $\mathrm{Hol}_{k}\left(\mathbb{P}^{1}, B U(n)\right)$.

In view of proposition 1.5 , it is sufficient to prove the following two theorems:

THEOREM 2.1. There is a natural homotopy equivalence

$$
H_{k}\left(\mathbb{P}^{1}, B U(n)\right) \simeq \mathcal{C}_{k, n}^{-} .
$$

THEOREM 2.2. The inclusion given by proposition 1.5

$$
\tilde{\Omega}_{k, n} \subset \mathcal{C}_{k, n}^{-}
$$

is a homotopy equivalence.

We begin with a proof of theorem 2.1.

Proof. We start by describing a model (up to homeomorphism) of $\operatorname{Hol}_{k}\left(\mathbb{P}^{1}, B U(n)\right)$. 
Let $V$ be an infinite dimensional complex vector space topologized as the union of its finite dimensional subspace. We take as our model for $B U(n)$ the Grassmannian $G r_{n}(V)$ of $n$-dimensional subspaces of $V . G r_{n}(V)$ is topologized as the limit

$$
G r_{n}(V)=\lim _{\longrightarrow} F r_{n}(F)
$$

where the limit is taken over finite dimensional subspaces $F$ of $V$.

Proposition 2.1. Let $V_{\infty} \subset V$ be a fixed $n$ - dimensional subspace. Define $\mathcal{M}_{k, n}$ to be the following moduli space:

$\mathcal{M}_{k, n}=\left\{\right.$ isomorphism classes of pairs $(E, j)$, where $E \rightarrow \mathbb{P}^{1}$ is a negative $n$ dimensional holomorphic bundle of Chern class $c_{1}(E)=-k$, and $j: E \hookrightarrow \mathbb{P}^{1} \times V$ is a holomorphic embedding of vector bundles taking the fiber at $\infty, E_{\infty}$ to $\left.V_{\infty}\right\}$

Then there is a natural set bijection

$$
h: \operatorname{Hol}_{k}\left(\mathbb{P}^{1}, G r_{n}(V)\right) \stackrel{\cong}{\longrightarrow} \mathcal{M}_{k, n} .
$$

Proof. Let $f \in \operatorname{Hol}_{k}\left(\mathbb{P}^{1}, G r_{n}(V)\right)$. The universal bundle $\zeta \rightarrow G r_{n}(V)$ is a holomorphic bundle embedded (holomorphically) in the trivial bundle $e: \zeta \hookrightarrow G r_{n}(V) \times V$. We define

$$
h(f)=\left(f^{*}(\zeta), g^{*}(e)\right) \in \mathcal{M}_{k, n} .
$$

The fact that $h$ is a bijection follows from the fact that a holomorphic bundle over $\mathbb{P}^{1}$ is (holomorphically) embeddable in a trivial bundle if and only if it is negative.

We give the set $\mathcal{M}_{k, n}$ the topology induced from $\operatorname{Hol}_{k}\left(\mathbb{P}^{1}, G r_{n}(V)\right)$ via the bijection $h$. Theorem 2.1 will then be a consequence of the following.

Proposition 2.2. The moduli spaces $\mathcal{C}_{k, n}^{-}$and $\mathcal{M}_{k, n}$ are homotopy equivalent.

Proof. We actually prove that the moduli spaces $\mathcal{M}_{k, n}$ and $\mathcal{C}_{k, n}^{-}$are both homotopy equivalent to an intermediate space $\mathcal{X}_{k, n}$ defined by

$\mathcal{X}_{k, n}=\left\{\right.$ isomorphism classes of pairs $(E, j, \theta)$, where $(E, j) \in \mathcal{M}_{k, n}$, and $\theta$ : $E_{\left.\right|_{D_{\infty}}} \rightarrow D_{\infty} \times V_{\infty}$ is a holomorphic trivialization $\}$.

Forgetting the trivialization $\theta$ defines a map

$$
\pi_{k, n}: \mathcal{X}_{k, n} \rightarrow \mathcal{M}_{k, n}
$$

Similarly the projection map $(E, j, \theta) \rightarrow(E, \theta)$ defines a map

$$
p_{k, n}: \mathcal{X}_{k, n} \rightarrow \mathcal{C}_{k, n}^{-}
$$

The following is rather technical, and so its proof will be delayed until the next section.

Lemma 2.3. a. The map $\pi_{k, n}: \mathcal{X}_{k, n} \rightarrow \mathcal{M}_{k, n}$ is a locally trivial fibration.

b. The map $p_{k, n}: \mathcal{X}_{k, n} \rightarrow \mathcal{C}_{k, n}^{-}$is a quasifibration.

Assuming the validity of this lemma for now, we can easily complete the proof of proposition 2.2. Notice that the fiber of the map $\pi_{k, n}$ is the space of (holomorphic) trivializations of $E_{\left.\right|_{D_{\infty}}}$, which is homeomorphic to $\operatorname{Hol}\left(D^{\infty}, G L(n, \mathbb{C})\right)$, which 
is contractible. Similarly the fiber of the map $p_{k, n}$ is the space of holomorphic bundle embeddings $E \hookrightarrow \mathbb{P}^{1} \times V$. Since every such embedding has image in a finite dimensional trivial subbundle, this space is given by the limit of the spaces of holomorphic embeddings of $E$ into finite dimensional trivial subbundles of $\mathbb{P}^{1} \times V$. Since $V$ is infinite dimensional this limiting space is contractible. Since $\pi_{k, n}$ and $p_{k, n}$ both have contractible fibers, then by the lemma they are homotopy equivalences. This implies $\mathcal{C}_{k, n}^{-}$and $\mathcal{M}_{k, n}$ are homotopy equivalent to each other.

This proves the proposition, and therefore completes the proof of theorem 2.1, modulo lemma 2.3.

We now proceed to prove theorem 2.2. We will give two proofs of this theorem. The first is homological in nature and will rely on the calculations of the homology of the spaces $F_{k, n}$ by Mitchell [7] and of spaces of holomorphic maps from $\mathbb{P}^{1}$ to Grassmannians by Mann and Milgram [6]. The second proof will be Morse theoretic in nature, and will rely on the dynamics of a flow of a natural $C^{*}$ - action on loop groups studied in [9]. This proof is more geometric in nature, and has the feature that it will give an alternative proof of Mann and Milgram's calculation of $H_{*}\left(H_{o l}\left(\mathbb{P}^{1}, G r_{n}(V)\right)\right)$.

Proof. (Homological Proof) Let $\beta: F_{k, n} \rightarrow \operatorname{Hol}_{k}\left(\mathbb{P}^{1}, G r_{n}(V)\right)$ be the composition

$$
\beta: F_{k, n} \cong \tilde{\Omega}_{k, n} \subset \mathcal{C}_{k, n}^{-} \simeq \operatorname{Hol}_{k}\left(\mathbb{P}^{1}, G r_{n}(V)\right) .
$$

Of course it suffices to prove that $\beta$ induces an isomorphism in homology.

Notice from the construction of the map $\beta$ that if one composes with the inclusion of holomorphic maps into smooth maps,

$$
F_{k, n} \stackrel{\beta}{\longrightarrow} \operatorname{Hol}_{k}\left(\mathbb{P}^{1}, B U(n)\right) \hookrightarrow \Omega_{k}^{2} B U(n) \simeq \Omega_{k} U(n) \simeq \Omega S U(n)
$$

then this map is homotopic to the inclusion of the Mitchell filtration $F_{k, n} \subset \Omega S U(n)$.

Also, consider the composition of $\beta$ with the map $\operatorname{Hol}_{k}\left(\mathbb{P}^{1}, G r_{n}(V)\right) \rightarrow B U(k)$ given by

$$
\delta: H o l_{k}\left(\mathbb{P}^{1}, G r_{n}(V)\right) \simeq \mathcal{C}_{k, n}^{-} \rightarrow G r_{k}\left(H_{+}^{n}\right)
$$

where the last map assigns to a loop $\gamma \in \Omega U(n)_{k}$ whose associated holomorphic bundle $E_{\gamma}$ is negative (and therefore lies in $\mathcal{C}_{k, n}^{-}$) the cokernel of the projection operator $p r_{\gamma}$ viewed as a subspace coker $\left(p r_{\gamma}\right) \subset H_{+}^{n}$. The composition

$$
F_{k, n} \stackrel{\beta}{\longrightarrow} \operatorname{Hol}_{k}\left(\mathbb{P}^{1}, G r_{n}(V)\right) \stackrel{\delta}{\longrightarrow} G r_{k}\left(H_{+}^{n}\right) \simeq B U(k)
$$

is homotopic to the map described in proposition 1.4. We therefore have the following homotopy commutative diagram:

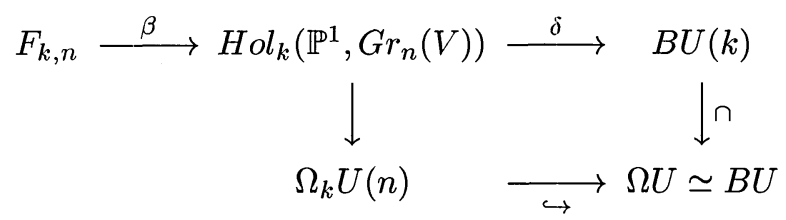

where the outer maps $\delta \circ \beta: F_{k, n} \rightarrow B U(k)$ and $F_{k, n} \rightarrow \Omega_{k} U(n)$ are the maps described in proposition 1.3. Now by this proposition, the composite map $F_{k, n} \rightarrow B U$ maps injectively in homology, with image equal to the intersection $H_{*}(\Omega U(n)) \cap H_{*}(B U(k)) \subset$ $H_{*}(B U)$. Thus by the commutativity of this diagram, the homomorphism

$$
\beta_{*}: H_{*}\left(F_{k, n}\right) \rightarrow H_{*}\left(\operatorname{Hol}_{k}\left(\mathbb{P}^{1}, G r_{n}(V)\right)\right)
$$


is injective. To prove surjectivity, we use a result of Mann and Milgram [7] that says the inclusion of the holomorphic maps into all smooth maps

$$
\operatorname{Hol}_{k}\left(\mathbb{P}^{1}, G r_{n}\left(\mathbb{C}^{m}\right)\right) \hookrightarrow \Omega_{k}^{2} G r_{n}\left(\mathbb{C}^{m}\right)
$$

induces an injection in homology for every Grassmannian $G r_{n}\left(\mathbb{C}^{m}\right)$. This implies that the map

$$
H_{*}\left(H o l_{k}\left(\mathbb{P}^{1}, G r_{n}(V)\right)\right)=H_{*}\left(H_{k} l_{k}\left(\mathbb{P}^{1}, B U(n)\right)\right) \rightarrow H_{*}\left(\Omega^{2} B U(n)\right)=H_{*}(\Omega U(n))
$$

is injective. But since the inclusion $H_{*}(\Omega U(n)) \rightarrow H_{*}(\Omega U) \cong H_{*}(B U)$ is injective, that means that the composition

$$
H_{*}\left(H o l_{k}\left(\mathbb{P}^{1}, G r_{n}(V)\right)\right) \rightarrow H_{*}(\Omega U(n)) \rightarrow H_{*}(B U)
$$

is injective. Also, by the commutativity of the diagram, we know that the image lies in the intersection $H_{*}(\Omega U(n)) \cap H_{*}(B U(k)) \subset H_{*}(B U)$. But as remarked above this intersection is the image of (and isomorphic to) $H_{*}\left(F_{k, n}\right)$. Hence both maps

$$
H_{*}\left(\operatorname{Hol}_{k}\left(\mathbb{P}^{1}, G r_{n}(V)\right)\right) \rightarrow H_{*}(\Omega U(n)) \cap H_{*}(B U(k))
$$

and

$$
H_{*}\left(F_{k, n}\right) \rightarrow H_{*}(\Omega U(n)) \cap H_{*}(B U(k))
$$

are isomorphsms. Thus by the commutativity of the above diagram

$$
\beta_{*}: H_{*}\left(F_{k, n}\right) \rightarrow H_{*}\left(\operatorname{Hol}_{k}\left(\mathbb{P}^{1}, G r_{n}(V)\right)\right)
$$

is an isomorphism. Now since both these spaces are known to be simply connected ([7], [6]), this implies $\beta$ is a homotopy equivalence.

Proof. (Morse theoretic proof) The second proof that the inclusion $\tilde{\Omega}_{k, n} \hookrightarrow \mathcal{C}_{k, n}^{-}$ is a homotopy equivalence is by studying the dynamics of a $\mathbb{C}^{*}$ - action on $\Omega U(n)$ described in [9].

To be more specific, consider the natural circle action on $H^{n}=L^{2}\left(S^{1}, \mathbb{C}^{n}\right)$. This action preserves the polarization and therefore induces an action on the Grassmannian $\operatorname{Gr}\left(H^{n}\right)$. It was shown in [9] that this action extends to a smooth action of the units in $\mathbb{C}$ lying in the unit disk

$$
\mathbb{C}_{\leq 1}^{*} \times G r^{(n)} \rightarrow G r^{(n)}
$$

and to all of $\mathbb{C}^{*}$ on the polynomial Grassmannian,

$$
\mathbb{C}^{*} \times G r_{0}^{(n)} \rightarrow G r_{0}^{(n)} .
$$

The induced flow given by the action of the reals was shown to be the gradient flow of the energy function on $\Omega U(n)$ :

$$
\begin{aligned}
E: \Omega U(n) & \rightarrow \mathbb{R} \\
\gamma & \rightarrow \frac{1}{4 \pi} \int_{0}^{2 \pi}\left|\gamma(t)^{-1} \gamma^{\prime}(t)\right|^{2} d t .
\end{aligned}
$$

The critical points of this action are homomorphisms $\lambda: S^{1} \rightarrow U(n)$, and hence the critical levels are indexed by the conjugacy class of the homomorphism; namely a 
partition $\bar{a}=\left(a_{1}, \cdots, a_{n}\right)$. We write $\lambda_{\bar{a}}$ to denote the critical level given by conjugates of the homomorphism

$$
z \rightarrow\left(z^{a_{1}}, \cdots, z^{a_{n}}\right) \in U(1) \times \cdots \times U(1) \subset U(n) .
$$

The resulting Morse decomposition of $\Omega U(n)=L G L(n, \mathbb{C}) / L^{+}$was studied in great detail in [9]. In particular it was shown that the stable and unstable manifolds of the critical levels $\lambda_{\bar{a}}$ are given by the orbits under the (left) action of $L^{-}$and $L^{+}$ respectively. (Here, as above, $L^{+}$denotes those loops that are boundary values of holomorphic maps of the disk $D_{0}$, and $L^{-}$denotes those loops that are boundary values of holomorphic maps of the disk around infinity, $D_{\infty}$.)

Recall that with respect to a generic metric, the unstable and stable manifolds of a Morse function intersect each other transversally. In this case the critical points have a partial ordering, where one says that $a \geq b$ if there is a flow line emanating from $a$ and converging to $b$. The resulting ordering of critical points of this flow on $\Omega U(n)$ was described in [9] in the following way.

Let $S \subset \mathbb{Z}$ be a set of integers whose symmetric difference with the nonnegative integers, $\mathbb{Z}^{+}$is finite. That is,

$$
S \Delta \mathbb{Z}^{+}=\left\{S-\mathbb{Z}^{+}\right\} \cup\left\{\mathbb{Z}^{+}-S\right\}
$$

is a finite set. The virtual cardinal of such a set $S, v \cdot c(S)$ is defined to be

$$
v . c(S)=\#\left\{S-\mathbb{Z}^{+}\right\}-\#\left\{\mathbb{Z}^{+}-S\right\} .
$$

A set $S$ with virtual cardinal $v . c(S)=k$ will be of type $n$, if for every $s \in S$,

$$
s+n \subset S .
$$

It was shown in [9] that the critical manifolds of the above flow when restricted to the component $\Omega_{k} U(n)$, are indexed by sets $S$ of type $n$ having virtual cardinal $=-k$. The relationship with the above indexing of critical points via conjugacy classes of homomorphisms $\lambda: S^{1} \rightarrow U(n)$, of winding number $k$, and thus partitions $\bar{a}=\left(a_{1}, \cdots, a_{n}\right)$, with $\sum_{i=1}^{n} a_{i}=k$, is given as follows.

Given such a partition $\bar{a}=\left(a_{1}, \cdots, a_{n}\right)$, then let $b_{i}=n a_{i}+i$, and let $S_{\bar{a}}$ be the type $n$ set genererated by $\left\{b_{1}, \cdots, b_{n}\right\}$. That is,

$$
S_{\bar{a}}=\bigcup_{q \in \mathbb{Z}^{+}}\left\{b_{1}+q n, b_{2}+q n, \cdots, b_{n}+q n\right\} .
$$

One reason that this indexing of the critical levels is useful, is that, as was observed in [9], every set $S$ with virtual cardinal $v \cdot c(S)=m$, can be written as an ordered sequence

$$
S=\left\{s_{-m}, s_{-m+1}, \cdots, s_{q}, \cdots\right\}
$$

with $s_{-m}<s_{-m+1}<\cdots, s_{q}, \cdots$, and $s_{m}=m$ for all $m$ sufficiently large. When written this way, the sets of virtual cardinal $k$ have a natural partial ordering given by

$$
S \geq S^{\prime} \quad \text { if } \quad s_{j} \leq s_{j}^{\prime} \quad \text { for all } j .
$$

The following was proved in [9]. 
LEMMA 2.4. The above partial ordering on the type $n$ sets $S$ of virtual cardinal $-k$ corresponds to the Morse - Smale partial ordering of the critical levels of the energy functional resticted to the component $\Omega_{k} U(n)$.

As we did with holomorphic bundles, we refer to a type $n$ set $S$ of virtual cardinal $-k$ as negative, if $S \subset \mathbb{Z}^{+}$. This terminology might be somewhat confusing, but we use it because in the definition $v . c(S)=\#\left\{S-\mathbb{Z}^{+}\right\}-\#\left\{\mathbb{Z}^{+}-S\right\}$, a negative set $S$ is one for which $\#\left\{S-\mathbb{Z}^{+}\right\}=0$ and hence $v \cdot c(S)=-\#\left\{\mathbb{Z}^{+}-S\right\}$.

For a given $k$ and $n$, define the type $n$ set of virtual cardinal $-k, S_{k}$, to be

$$
S_{k}=\{k, k+1, k+2, \cdots\} \text {. }
$$

Write the integer $k$ in the form $k=m n+i$, where $0 \leq i \leq n-1$. Then it is seen from the definition that the partition that $S_{k}$ corresponds to is $(m, m, \cdots, m, m+1, \cdots, m+1)$, where there are $n-i$ copies of $m$ and $i$ copies of $m+1$ in this partition of $k$.

$S_{k}$ therefore corresponds to the homomorphism

$$
\begin{aligned}
\lambda_{k}: S^{1} & \rightarrow U(1) \times \cdots \times U(1) \subset U(n) \\
z & \rightarrow\left(z^{m}, \cdots, z^{m}, z^{m+1}, \cdots, z^{m+1}\right)
\end{aligned}
$$

or, equivalently to the holomorphic bundle given by the direct sum

$$
\lambda_{i}=\oplus_{n-i} O(-m) \quad \ominus \quad \oplus_{i} O(-(m+1)) .
$$

The following is an exercise with the above definitions.

LEMMA 2.5. The sets $S_{k}$ are minimal in the sense that if $S$ is a type $n$ set of virtual cardinal $-k$ with

$$
S_{k} \geq S
$$

then $S=S_{k}$. Furthermore, a type $n$ set $S$ of virtual cardinal $-k$ satisfies

$$
S \geq S_{k}
$$

if and only if $S$ is negative.

This leads quite quickly to the following result.

Proposition 2.6. The moduli space $\mathcal{C}_{k, n}^{-}$, when viewed as a subspace of $\Omega U(n)_{k}$, is equal to the union of the stable manifolds of the critical levels indexed by type $n$ sets $S$ of virtual cardinal $-k$, satisfying $S \geq S_{k}$. That is, $\mathcal{C}_{k, n}^{-}$is the closure of the stable manifold of the critical level consisting of homomorphisms conjugate to $\lambda_{k}$, as defined above.

Proof. As described above, the stable manifold of the critical level of the space of homomorphisms conjugate to $\lambda_{\bar{a}}$ is given by the orbit under the left action of $L^{-}(G L(n, \mathbb{C}))$. Now the Bruhat factorization (see [9]) of a loop says that every loop $\gamma \in L G L(n, \mathbb{C})$ has a decomposition as

$$
\gamma=\gamma_{-} \cdot \lambda \cdot \gamma_{+}
$$

where $\gamma_{-} \in L^{-}$and $\gamma_{+} \in L^{+}$, and $\lambda: S^{1} \rightarrow U(n)$ is a homomorphism. This decomposition is unique up to the conjugacy class of $\lambda$. The corresponding holomorphic 
bundle $E_{\gamma}$ is isomorphic to $E_{\lambda}$ which is a direct sum of line bundles, and hence gives the Grothendieck decomposition. This then says that under the left action of $L^{-}$, the Grothendieck type of the corresponding holomorphic bundle is preserved. Thus the stable manifold of a critical set $\lambda_{\bar{a}}$ is exactly the space of loops $\gamma$ whose corresponding holomorphic bundles $E_{\gamma}$ are isomorphic to the direct sum of line bundles given by the partition $\vec{a}$. The proposition then follows from lemma 2.4 .

The action of $L^{+} G L(n, \mathbb{C})$ on the polynomial loop group $\Omega_{p o l} U(n)_{k}$ was studied in detail in [9] as well. As mentioned above, the orbits of this action give the unstable manifolds of the flow of energy functional, when restricted to the polynomial loop group. The following result can be viewed as the dual of proposition 2.6.

Proposition 2.7. The space $\tilde{\Omega}_{k, n}$, when viewed as a subspace of $\Omega_{p o l} U(n)_{k}$, is equal to the union of the unstable manifolds of the critical levels indexed by type $n$ sets $S$ of virtual cardinal $-k$, satisfying $S \geq S_{k}$.

Theorem 2.2 now follows from propositions 2.5 and 2.6 and standard Morse theory arguments, using the fact that the energy functional on $\Omega U(n)$ satisfies the Palais Smale condition.

Now that we have theorem 2 , we have identified the homotopy type of the holomorphic mapping space, $H_{o l}\left(\mathbb{P}^{1}, B U(n)\right)$ in terms of the Mitchell filtration, $F_{k, n}$. By taking the limit over the rank $n$ and using proposition 1.4, we get a homotopy equivalence

$$
\phi_{k}: \operatorname{Hol}_{k}\left(\mathbb{P}^{1}, B U\right) \stackrel{\simeq}{\longrightarrow} B U(k) .
$$

This is the assertion of theorem 1 .

3. Proof of Lemma 2.3. In the last section we completed the proofs of theorems 1 and 2 modulo a technical result (lemma 2.3) establishing that certain maps are (quasi)fibrations. We will prove this lemma in this section.

Proof. ( lemma 2.3 part (a)).

Recall that the space $\mathcal{X}_{k, n}$ is defined by $\mathcal{X}_{k, n}=$ isomorphism classes of triples $(E, j, \theta)$, where $(E, j) \in \mathcal{M}_{k, n}$, and $\theta: E_{\left.\right|_{D_{\infty}}} \rightarrow D_{\infty} \times V_{\infty}$ is a holomorphic trivialization\},

where $V$ is an infinite dimensional complex vector space (topologized as the limit of its finite dimensional subspaces), and $V_{\infty} \subset V$ is a fixed $n$ - dimensional subspace. Also recall that $\mathcal{M}_{k, n}$ is naturally homeomorphic to the holomorphic mapping space $\mathrm{Hol}_{k}\left(\mathbb{P}^{1}, G r_{n}(V)\right.$ ) (propositon 2.1). It is therefore clear that we can identify the elements of $\mathcal{X}_{k, n}$ as a set with the family of commutative diagrams,

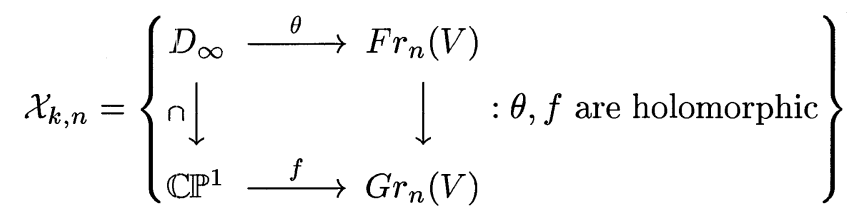

where $F_{n}(V)$ is the usual $G L(n, \mathbb{C})$ principal frame bundle over the Grassmannian $G r_{n}(V)$. This in turn establishes the topology on $\mathcal{X}_{k, n}$, where the above family of 
commutative diagrams is topologized as a subspace of the obvious product of mapping spaces (given the compact open topology).

Observe that $\mathcal{M}_{k, n}$ is the limit of the connected complex manifolds

$$
\mathcal{M}_{k, n}^{m}=H o l_{k}\left(\mathbb{C P}^{1}, G r_{n}\left(\mathbb{C}^{n+m}\right)\right) .
$$

We now define $\mathcal{X}_{k, n}^{m}$ in the same way as $\mathcal{X}_{k, n}$, except the vector space $V$ is replaced by $\mathbb{C}^{n+m}$. Namely,

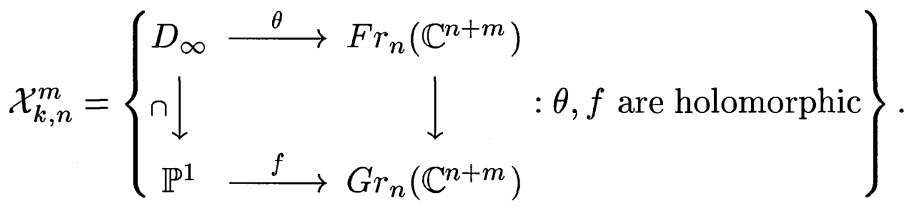

We now prove that

$$
\pi_{m}: \mathcal{X}_{k, n}^{m} \rightarrow \mathcal{M}_{k, n}^{m}
$$

is a locally trivial fibration. This is enough to conclude that $\pi$ is a locally trivial fibration.

To do this, let $f: \mathbb{P}^{1} \rightarrow G r_{n}\left(\mathbb{C}^{n+m}\right)$ lie in $\mathcal{M}_{k, n}^{m}$, and let $U$ be an open neighborhood of $f$. Recall that $\mathcal{M}_{k, n}^{m}$ is a complex manifold of dimension $k(n+m)$, so we can take $U$ to be a holomorphic disk of that dimension. Consider the adjoint of the inclusion map

$$
\iota: U \times \mathbb{P}^{1} \rightarrow G r_{n}\left(\mathbb{C}^{n+m}\right) .
$$

This is a holomorphic map. When restricted to the holomorphic disk $U \times D_{\infty}, \iota^{*}\left(E_{n}\right)$ is holomorphically trivial. Here $E_{n} \rightarrow G r_{n}\left(\mathbb{C}^{n+m}\right)$ is the universal bundle. Let

$$
\Psi: U \times D_{\infty} \times \mathbb{C}^{n} \stackrel{\cong}{\longrightarrow} \iota^{*}\left(E_{n}\right)_{\left.\right|_{U \times D_{\infty}}}
$$

be such a trivialization. For each $(x, y) \in U \times D_{\infty}$,

$$
\Psi(x, y): \mathbb{C}^{n} \cong \iota(x, y) \subset \mathbb{C}^{n+m}
$$

is a linear embedding. This gives a lift

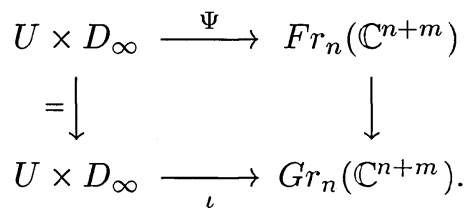

By definition, this defines a section $\Psi: U \rightarrow \mathcal{X}_{k, n}^{m}$. To get an induced trivialization, let $\gamma: D_{\infty} \rightarrow G L(n, \mathbb{C})$ be any holomorphic map, and define

$$
\Psi_{\gamma}: U \times D_{\infty} \rightarrow F r_{n}\left(\mathbb{C}^{n+m}\right)
$$

by

$$
\Psi_{\gamma}(x, y)=\Psi(x, y) \circ \gamma(y): \mathbb{C}^{n} \stackrel{\gamma(y)}{\longrightarrow} \mathbb{C}^{n} \stackrel{\Psi(x, y)}{\longrightarrow} \iota(x, y) \subset \mathbb{C}^{n+m}
$$


This then defines

$$
\bar{\Psi}: U \times D_{\infty} \times \operatorname{Hol}\left(D_{\infty}, G L(n, \mathbb{C})\right) \rightarrow F r_{n}\left(\mathbb{C}^{n+m}\right)
$$

or, equivalently,

$$
\bar{\Psi}: U \times \operatorname{Hol}\left(D_{\infty}, G L(n, \mathbb{C})\right) \rightarrow \pi_{m}^{-1}(U)
$$

given by $\bar{\Psi}(x, \gamma)=$

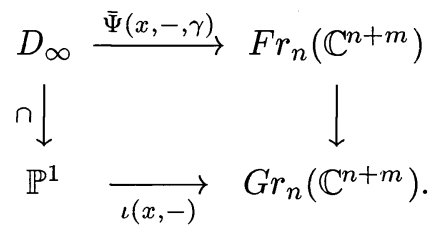

This gives our local trivialization of $\pi_{m}$ and therefore completes the proof of part (a) of lemma 2.3.

Proof. (lemma 2.3 part (b)). The first step in proving that $p_{k, n}$ (which we abbreviate simply as $p$ ) is a quasifibration is to prove that it is continuous. To do this we keep the notation as above.

Recall that the moduli space $\mathcal{C}_{k, n}^{-}$is a subspace of

$$
\Omega U(n) \cong L G L(n, \mathbb{C}) / L^{+} G L(n, \mathbb{C}) .
$$

Now given a point $(f, \theta) \in \mathcal{X}_{k, n}$ then there exists a neighborhood $\tilde{\mathcal{U}}$ around it in $\mathcal{X}_{k, n}$ so that $\left.p\right|_{\tilde{\mathcal{U}}}$ can be factored as

$$
\tilde{\mathcal{U}} \stackrel{\Theta}{\longrightarrow} L G L(n, \mathbb{C}) \rightarrow L G L(n, \mathbb{C}) / L^{+} G L(n, \mathbb{C})
$$

and hence we only need to prove that the map $\Theta$ is continuous.

We choose $\tilde{\mathcal{U}}=\pi^{-1}(\mathcal{U})$ as above, then with respect to the above local trivialization, $\Theta$ has the formula

$$
\begin{gathered}
\Theta: \mathcal{U} \times \operatorname{Hol}\left(D_{\infty}, G L(n, \mathbb{C})\right) \rightarrow L G L(n, \mathbb{C}) \\
\Theta(f(z), \tau(z))=\tau(z) \cdot P(z) .
\end{gathered}
$$

This is just the attaching map induced by the canonical trivialization given by the local section $\psi$. Clearly $\Theta$ is continuous.

Finally, we need to show that $p$ is a quasifibration. To prove this we will use the following proposition about quasifibrations taken directly from [12], which in turn is a consequence of theorem 2.15 in the classical paper by A. Dold and R. Thom [13].

Proposition 3.1. . Let $B$ be a space filtered by subspaces

$$
B_{1} \hookrightarrow B_{2} \hookrightarrow \cdots \hookrightarrow B_{n} \hookrightarrow \cdots \hookrightarrow B
$$

with $B=\bigcup_{n} B_{n}$ having the topology of the union. Suppose

$$
p: E \longrightarrow B
$$


is a surjective map, and that there are trivializations on the strata, $E_{n+1}-E_{n} \cong$ $\left(B_{n+1}-B_{n}\right) \times F$, where $E_{n}=E_{\left.\right|_{B_{n}}}$. Suppose also that every $B_{n}$ is a strong deformation retract of a neighborhood $V_{n} \subset B_{n+1}$, with retraction $r_{n}: V_{n} \rightarrow B_{n}$. Suppose furthermore that the retractions are covered by maps $\tilde{r}_{n}$,

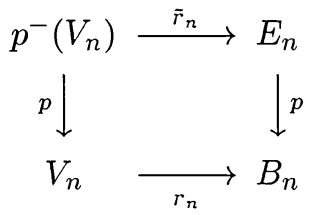

that induce homotopy equivalences on the fibers. Then $p$ is a quasifibration.

Now in order to apply this proposition in our situation we consider the Birkhoff and Bruhat factorization theorems proved in chapter 8 of [9].

For a given $\gamma \in \mathcal{C}_{k, n}^{-} \subset \Omega U(n)$, write $\gamma=(E, \theta)$ as above. Then the proofs of these theorems give a canonical isomorphism with its Grothendieck decomposition,

$$
E \cong \mathcal{O}\left(k_{1}\right) \oplus \cdots \oplus \mathcal{O}\left(k_{n}\right)
$$

Moreover this isomorphism clearly extends over the neighborhood $U_{\gamma}$ of $\gamma$ in $\mathcal{C}_{k, n}^{-}$ in which the Grothendieck type of the holomorphic bundle is constant. Hence when restricted to this neighborhood, the fiber of $p_{\left.\right|_{U_{\gamma}}}: \mathcal{X}_{k, n_{\left.\right|_{U_{\gamma}}}} \rightarrow U_{\gamma}$ is canonically identified with the space of linear holomorphic embeddings of $\mathcal{O}\left(k_{1}\right) \oplus \cdots \oplus \mathcal{O}\left(k_{n}\right)$ into the infinite dimensional trivial bundle $\mathbb{P}^{1} \times V, \operatorname{Emb}\left(\mathcal{O}\left(k_{1}\right) \oplus \cdots \oplus \mathcal{O}\left(k_{n}\right), \mathbb{P}^{1} \times V\right)$.

Now in section 8.4 of [9] a "Bruhat" stratification of $\Omega U(n)$ is studied, which as observed earlier coincides with the Morse stratification of the energy functional

$$
E: \Omega U(n) \rightarrow \mathbb{R}
$$

described in section 2 above. As seen there (proposition 2.8), this stratification restricts to a Morse stratification of $\mathcal{C}_{k, n}^{-}$, where the strata consist of those loops $\gamma=(E, \theta)$, where $E$ has fixed Grothendieck type. Thus

$$
p: \mathcal{X}_{k, n} \longrightarrow \mathcal{C}_{k, n}^{-}
$$

is surjective, and has canonical local trivializations on the strata. Again, the fiber of $p$. on the stratum corresponding to loops $\gamma=(E, \theta)$ with $E \cong \mathcal{O}\left(k_{1}\right) \oplus \cdots \oplus \mathcal{O}\left(k_{n}\right)$ is given by the space of linear embeddings $\operatorname{Emb}\left(\mathcal{O}\left(k_{1}\right) \oplus \cdots \oplus \mathcal{O}\left(k_{n}\right), \mathbb{P}^{1} \times V\right)$. This space is nonempty because the Grothendieck type is negative, and it is contractible because $V$ is infinite dimensional.

As studied in section 2 above, these strata are partially ordered in a way corresponding to the Morse - Smale partial ordering of the critical levels of the energy functional restricted to $\mathcal{C}_{k, n}^{-}$(lemma 2.6). This then defines a partially ordered filtration of $\mathcal{C}_{k, n}^{-}$, indexed by the Grothendieck types, where $p$ restricted to the strata has canonical trivialization. Moreover standard Morse theory tells us that the inclusion of one filtration another has a neighborhood deformation retract, where the retractions are given by following flow lines of the energy functional. These retractions are then clearly covered by maps of the restrictions of $\mathcal{X}_{k, n}$ to these spaces. These are again defined by following flow lines. The fact that these maps induce homotopy equivalences on fibers follows from the fact that all the fibers (i.e the embedding spaces described above) are contractible. Hence by proposition 3.1 the map $p: \mathcal{X}_{k, n} \rightarrow \mathcal{C}_{k, n}^{-}$ is a quasifibration, as claimed. This completes the proof of lemma 2.3. 


\section{REFERENCES}

[1] M. F. AтাYah, Bott Periodicity and the index of elliptic operators, Quart. Jour. Math, 19 (1968), pp. 113-140.

[2] M. F. ATIYAH, Instantons in two and four dimensions, Comm. Math. Phys., 93 (1984), pp. 437-451.

[3] S. Donaldson, Instantons and Geometric Invariant theory, Comm. Math. Phys., 93 (1984), pp. 456-460.

[4] A. GRothendieck, Sur la classification des fibres holomorphes sur la sphere de Riemann, Amer. Jour. Math., 79 (1957), pp. 121-138.

[5] F. KIRwan, Geometric Invariant Theory and the Atiyah Jones Conjecture, Proc. S. Lie Mem. Conf., Scand. Univ. Press, 1994, pp. 161-188.

[6] B. Mann And R. J. Milgram, Some spaces of holomorphic maps to complex Grassmann manifolds, J. Diff. Geo., 33 (1991), pp. 301-324.

[7] S. Mitchell, The filtration of the loops on $S U(n)$ by Schubert varieties, Math Zeit., 193 (1986), pp. 347-362.

[8] W. RICHTER, Splitting of loop groups, To appear.

[9] A. Pressley and G. Segal, Loop Groups, Oxford Math. Monographs, Clarendon Press, 1986.

[10] M. SANDERS, Classifying spaces and Dirac operators coupled to instantons, Trans. of AMS, 347 (1995), pp. 4037-4072.

[11] G. B. SEGAL, Loop groups and harmonic maps, in London Math Soc Lect. Notes 139, 1989, pp. 153-164.

[12] M. Aguilar and C. Prieto, Quasifibrations and Bott periodicity, To appear.

[13] A. DOLD AND R. THOM, Quasifaserungen und unendliche symmetrische produkte, Annals of Math., 67 (1958), pp. 239-281. 